\title{
ER-localized auxin transporter PIN8 regulates auxin homeostasis and male gametophyte development in Arabidopsis
}

\author{
Zhaojun Ding ${ }^{1,2}$, Bangjun Wang ${ }^{3}$, Ignacio Moreno ${ }^{4}$, Nikoleta Dupláková ${ }^{5}$, Sibu Simon ${ }^{1}$, Nicola Carraro 6 , \\ Jesica Reemmer ${ }^{6}$, Aleš Pěnčík ${ }^{7}$, Xu Chen ${ }^{1}$, Ricardo Tejos ${ }^{1}$, Petr Skůpa ${ }^{5}$, Stephan Pollmann ${ }^{8}$, Jozef Mravec ${ }^{1}$, \\ Jan Petrášek ${ }^{5}$, Eva Zažímalová5 ${ }^{5}$ David Honys ${ }^{5}$, Jakub Rolčík ${ }^{7}$, Angus Murphy ${ }^{6}$, Ariel Orellana ${ }^{4}$, \\ Markus Geisler ${ }^{3}$ \& Jiř í Friml1,9
}

Auxin is a key coordinative signal required for many aspects of plant development and its levels are controlled by auxin metabolism and intercellular auxin transport. Here we find that a member of PIN auxin transporter family, PIN8 is expressed in male gametophyte of Arabidopsis thaliana and has a crucial role in pollen development and functionality. Ectopic expression in sporophytic tissues establishes a role of PIN8 in regulating auxin homoeostasis and metabolism. PIN8 co-localizes with PIN5 to the endoplasmic reticulum (ER) where it acts as an auxin transporter. Genetic analyses reveal an antagonistic action of PIN5 and PIN8 in the regulation of intracellular auxin homoeostasis and gametophyte as well as sporophyte development. Our results reveal a role of the auxin transport in male gametophyte development in which the distinct actions of ER-localized PIN transporters regulate cellular auxin homoeostasis and maintain the auxin levels optimal for pollen development and pollen tube growth.

\footnotetext{
${ }^{1}$ Department of Plant Systems Biology, VIB and Department of Plant Biotechnology and Genetics, Ghent University, B-9052 Gent, Belgium. ${ }^{2}$ The Key Laboratory of Plant Cell Engineering and Germplasm Innovation, Ministry of Education, School of Life Sciences, Shandong University, Shanda Nanlu 27 , Jinan 250100, China. ${ }^{3}$ Department of Biology, Plant Biology, University of Fribourg, 1700 Fribourg, Switzerland. ${ }^{4}$ Center for Genome Regulation, Center of Plant Biotechnology, Facultad de Ciencias Biologicas, Universidad Andres Bello, Santiago, Chile. ${ }^{5}$ Institute of Experimental Botany ASCR, 16500 , Praha 6, Czech Republic. ${ }^{6}$ Department of Horticulture, Purdue University, West Lafayette, Indiana, USA. ${ }^{7}$ Laboratory of Growth Regulators, Centre of the Region Haná for Biotechnological and Agricultural Research, Faculty of Science, Palacký University, 78371 Olomouc, Czech Republic. ${ }^{8}$ Ruhr-Universität Bochum, Lehrstuhl für Pflanzenphysiologie, 44801 Bochum, Germany. ${ }^{9}$ Department of Functional Genomics and Proteomics, Faculty of Science, and CEITEC, Masaryk University, Kamenice 5, CZ-62500 Brno, Czech Republic. Correspondence and request for materials should be addressed to J.F. (e-mail: jiri.friml@psb.vib-ugent.be).
} 
$\mathrm{T}$ he plant hormone auxin has a crucial role in plant development ${ }^{1-6}$. On account of its differential distribution within plant tissues, it acts as a versatile coordinative signal mediating a multitude of processes, including female gametophyte patterning, embryogenesis, organogenesis, meristem activity, growth responses to environmental stimuli and others ${ }^{7-10}$. High auxin concentration in germinating pollen and active auxin responses in developing pollen ${ }^{11-13}$ implicated auxin also in male gametophyte development and function, but its role there remained elusive.

A crucial aspect of auxin action is its graded distribution (auxin gradients) that depends on local auxin biosynthesis ${ }^{14-16}$ and directional, intercellular auxin transport ${ }^{7,17,18}$. The polar auxin transport has an essential role in most auxin-regulated processes and is mediated by auxin influx proteins from the AUX1/LAX family ${ }^{19}$, by PIN auxin efflux proteins ${ }^{20}$ and by homologues of the $\mathrm{ABCB}$ multiple drug resistance transporters ${ }^{21,22}$. These auxin transporters typically localize to the plasma membrane (PM) and facilitate auxin flow across the membrane in and out of the cell ${ }^{23}$. Recently, the Arabidopsis thaliana PIN5 auxin transporter has been shown to localize to the endoplasmic reticulum (ER) and its activity is important for the regulation of auxin metabolism ${ }^{24}$. These findings suggest the existence of auxin transport across the ER membrane, although a demonstration of such transport has not been provided yet and its role in auxin biology and plant development is still largely unclear.

Here we identify and functionally characterize PIN8, an auxin transporter preferentially expressed in male gametophyte. PIN8 transports auxin across the ER membrane and antagonistically to PIN5 regulates intracellular auxin homoeostasis and metabolism for male gametophyte function.

\section{Results}

PIN8 is expressed and functions in the male gametophyte. To assess a potential role for the auxin transport in male gametophyte development, we examined the expression patterns of PIN auxin transporters by using available transcriptome data ${ }^{25}$. Among the eight members of the PIN protein family in the Arabidopsis genome, a so-far uncharacterized member PIN8 was the only one predominantly expressed in pollen (Supplementary Fig. S1a). Reverse transcription PCR confirmed the highly enriched expression of PIN8 in reproductive tissues (Supplementary Fig. S1b) and lines comprising the PIN8 coding region fused with green fluorescent protein (GFP) under the control of its native promoter (PIN8::PIN8GFP) showed strong signal in developing and germinating pollen (Supplementary Fig. S1a).

To analyse the potential role of PIN8 in pollen development, we isolated two insertion mutant alleles, pin8-1 and pin8-2, disrupting the first and fifth exon of the PIN8 gene, respectively (Supplementary Fig. S1c,d). No obvious phenotypic defects were observed in seedlings and adult plants in both alleles (data not shown), which was consistent with very low PIN8 expression in most sporophytic tissues. However, pin 8 mutants showed a decreased transmission ability through male gametophyte (38\%) for pin8-1 versus the expected $50 \%$ for the wild-type (Col-0) pollen (Fig. 1a). The complementation of this phenotype confirmed that the described pollen transmission defects are due to the loss of PIN8 function (Supplementary Fig. S2). 4',6-Diamidino-2-phenylindole (DAPI) staining of pollen revealed a high frequency of aborted and misshaped pollen grains in pin8 (Fig. 1b,c, Supplementary Table S1) and transmission electron microscopy analyses showed that pin 8 mutant pollen has a reduced density of rough ER (10-15\%, $n>150$ pollen grains) in comparison to the wild-type pollen (Fig. 1d,e). Next, we examined transgenic lines overexpressing PIN8 under strong pollenspecific promoter LAT52 (ref. 24). LAT52::PIN8 also showed strongly reduced transmission through the male gametophyte (below 10\% for LAT52::PIN8 versus expected 50\% for the wild-type control; Fig. 1a and Supplementary Fig. S1e). In addition, in vitro
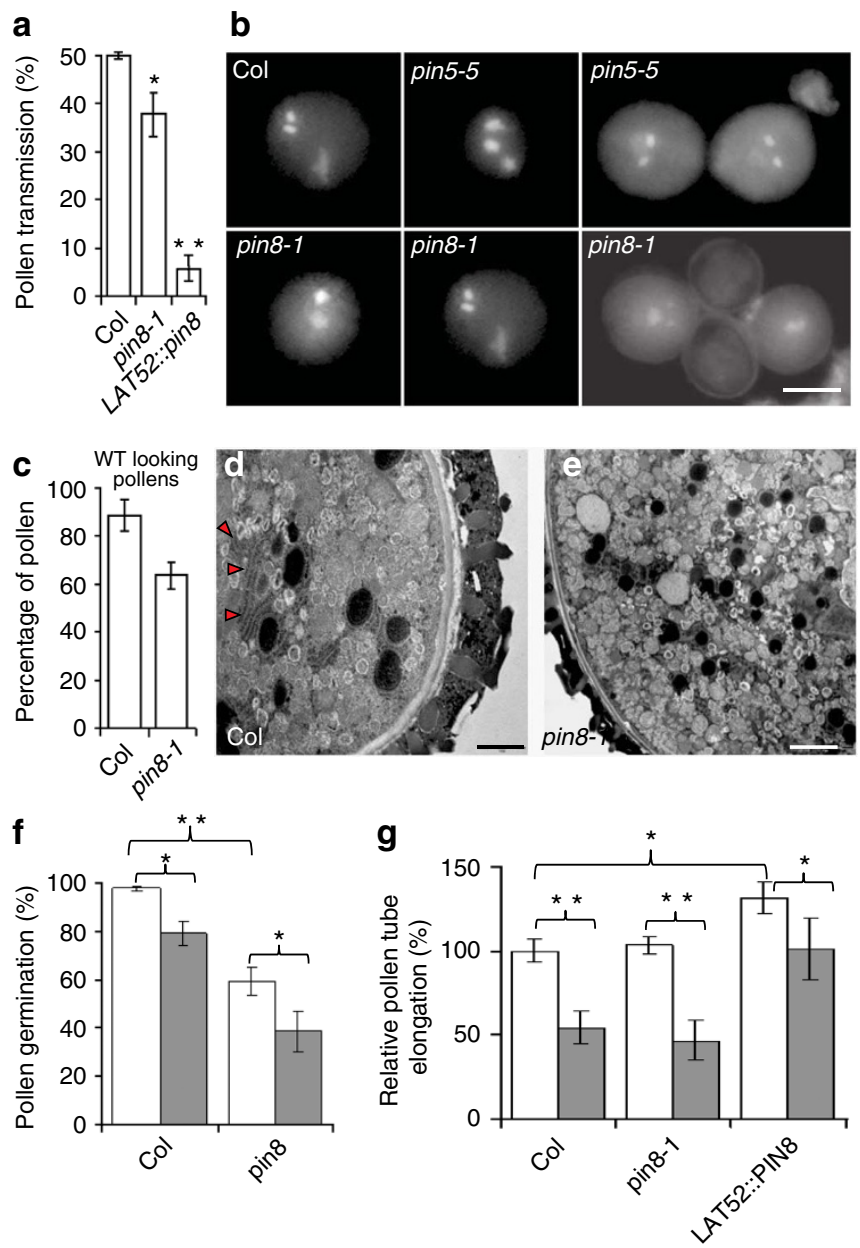

Figure 1 | PIN8 is involved predominantly in the male gametophyte development. (a) The pin8 mutant and the LAT52::PIN8 line showed reduced pollen transmission ability. Error bars represent the standard error of more than ten independent crosses (Student's $t$-test, ${ }^{\star} P<0.05$ ). $(\mathbf{b}, \mathbf{c})$ DAPI staining analysis showing defects in the morphology of pin8 and pin 5 mutant pollen. Both mutants showed distorted and/or misplaced male germ unit and less frequently also exhibit pollen mitosis defects. Sometimes, collapsed pollen grains were observed. Scale bar, $10 \mu \mathrm{m}$. Error bars represent the standard error of more than 23 independent plants (Student's t-test, $\left.{ }^{\star \star} P<0.01\right)$. (d,e) Typical ER clusters in wild-type (WT) pollen (d) were not observed in 10-15\% pin8 pollen (observed 150 pollen grains) (e) by transmission electron microscope analysis. Scale bar, $10 \mu \mathrm{m}$. Red arrows mark ER clusters. (f) pin8 (2256 pollen were analysed) shows reduced in vitro pollen germination abilities (2,814 Col pollens were analysed as the control) and increased sensitivity to auxin treatment (100 nM NAA) with a $35 \%$ reduction of pollen germination in pin8 (5,017 pollens were analysed) compared with the $19 \%$ reduction in Col (1,131 pollens were analysed). Error bars represent the standard error of more than ten independent plants (Student's $t$-test, ${ }^{\star} P<0.05$; ${ }^{\star \star} P<<0.01$ ). White and grey columns represent without and with NAA treatment, respectively. (g) Overexpression of PIN8 in pollen in the LAT52 ::PIN8 line strongly increased the resistance (with a $24 \%$ inhibition of pollen tube length compared with the $46 \%$ inhibition in Col) of in vitro pollen germination to NPA (100 $\mu \mathrm{M}$ NPA) treatment. Error bars represent the standard error of more than six independent plants (Student's t-test, $\left.{ }^{\star} P<0.05 ;{ }^{\star \star} P<0.01\right)$. White and grey column represent without and with NPA treatment, respectively, in Col, pin8 or LAT52::PIN8.

pollen germination assays revealed a decreased pollen germination in pin 8 (40\% decrease, Fig. 1f) and increased pollen tube elongation in LAT52::PIN8 lines (30\% increase, Fig. 1g) with a normal pollen 
tube morphology (Supplementary Fig. S1f). Thus, the loss-offunction and ectopic expression analyses revealed a specific role of PIN8 in male gametophyte development and function.

As PIN8 is a member of the auxin transporter family, it is presumably involved in auxin transport. However, a role of auxin transport in male gametophyte development and function is far from being clear. To address this issue, we pharmacologically manipulated auxin levels and auxin transport during pollen germination and pollen tube growth. Auxin treatments $(100 \mathrm{nM} \alpha$-naphthaleneacetic acid (NAA)) reduced the pollen germination rate in wild type (19\% reduction) and pin8-1 pollen showed increased sensitivity to such auxin treatment (35\% reduction) (Fig. 1f). Furthermore, the auxin transport inhibitor 1-N-naphthylphthalamic acid (NPA) strongly inhibited pollen tube growth and, notably, LAT52::PIN8 pollen showed increased resistance to this NPA effect (Fig. 1g). The inhibition of the in vitro pollen tube growth by NPA was apparent only at high concentrations $(100 \mu \mathrm{M})$ indicating that it might occur through APM1-regulated functions of PIN and $\mathrm{ABCB}$ auxin transport proteins, as APM1 is the target at high concentrations of NPA treatment ${ }^{26,27}$. The change in sensitivity in pollen tubes overexpressing auxin transport-related protein PIN8 suggests that it is auxin transport-related effect of NPA that affects pollen tube growth. Collectively, these findings hint at an important role of auxin and auxin transport for male gametophyte function and identified the pollen-specific putative auxin transporter PIN8 as an important factor regulating these processes.

PIN8 overexpression affects many aspects of plant growth and development. To further characterize the function of PIN8, we generated lines overexpressing PIN8 in sporophytic tissues under the control of the strong cauliflower mosaic virus $35 \mathrm{~S}$ promoter (PIN8OX). PIN8OX lines flowered much earlier than wild-type plants (Fig. 2a,b), showed enhanced leaf margin serration, especially under short-day growth conditions (Fig. 2c) and had much longer hypocotyls than the control (Fig. 2d,e), which can be attributed to the increased cell elongation (Fig. 2f and Supplementary Table S2). All these plant growth and development phenotypes in PIN8OX suggest a capacity of PIN8 to influence different aspects of plant development, preferentially those where auxin is involved.

PIN8 and PIN5 localize to the ER. In Arabidopsis, the PIN auxin transporter family can be divided in two subclades: one represented by PIN1, PIN2, PIN3, PIN4 and PIN7, which all localize to the $\mathrm{PM}^{23}$, and the other subclade represented by PIN5, PIN8 and possibly PIN6, which are characterized by a reduced middle hydrophilic loop ${ }^{23}$ and for which PIN5 has been shown to localize to the ER in Arabidopsis ${ }^{24}$. To investigate the subcellular localization of PIN8, we examined Arabidopsis transgenic lines expressing a functional PIN8 ::PIN8-GFP construct. In PIN8 ::PIN8-GFP (Supplementary Fig. S2), a specific intracellular signal was observed only in pollen and growing pollen tubes (Fig. 3a-c and Supplementary Fig. S1a) consistent with the PIN8 expression data (Supplementary Fig. S1a). The PIN8-GFP-expressing cells showed intracellular signals, but no localization to the PM (Fig. 3a-c). A pronounced co-localization with the ER-tracer dye ${ }^{24}$ suggested the association of PIN8-GFP with the ER (Fig. 3a-c).

To examine the PIN8 localization in more detail and in different sporophytic cell types, we ectopically expressed PIN8 fused with GFP under the control of $35 \mathrm{~S}$ promoter. Similar to what was observed in pollen, we detected a consistent, intracellular PIN8-GFP signal co-localized with ER markers, such as BIP2 (ref. 28) in root cells (Fig. 3d-f, p). On the other hand, we did not observe any co-localization of PIN8 with endosomes or Golgi apparatus (Fig. 3g-i, p and Supplementary Fig. S3) and also we did not observe any co-localization with labelled PM (Fig. 3j-l, p). Furthermore, PIN8 co-localized with PIN5 at the ER (Fig. 3m-p) and this
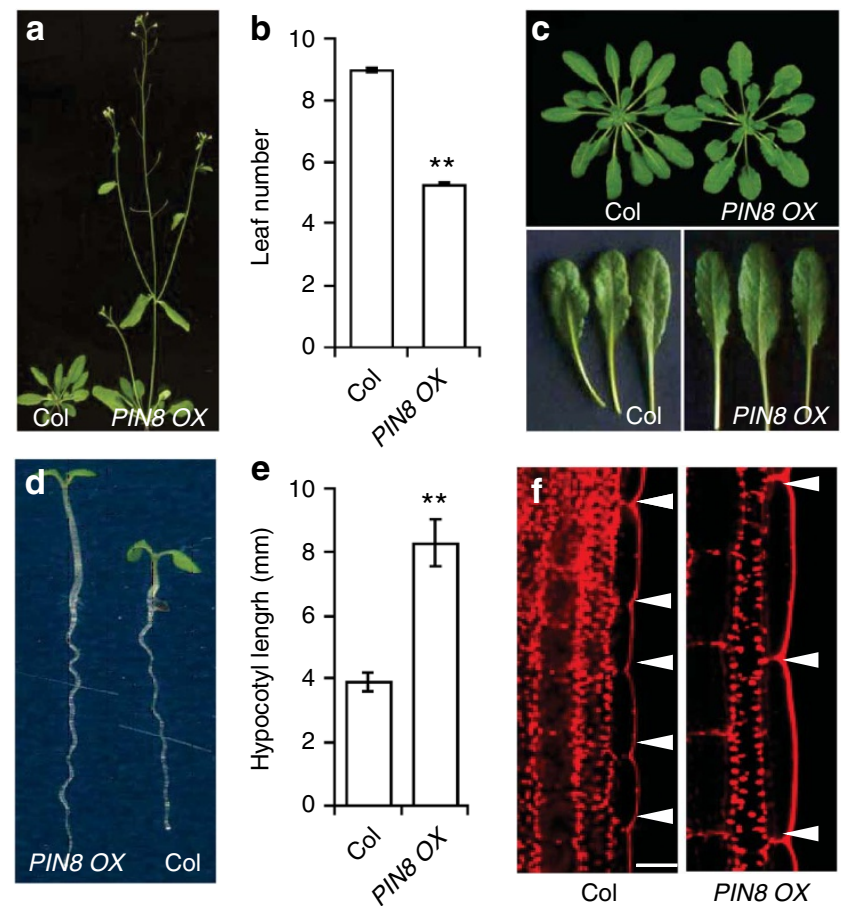

Figure 2 | PIN80X lines showed defects in many aspects of plant growth and development. (a,b) PIN8OX lines flower earlier than the wild-type controls measured under long-day growth conditions. Error bars represent the standard error of the mean from three independent experiments ( $n=48$, Student's $t$-test, ${ }^{\star \star} P<0.01$ ). (c) PIN80X lines showed strongly enhanced leaf margin serration under short-day growth conditions. (d,e) PIN80X lines showed long hypocotyls compared with the control. Hypocotyl length was measured with 5-day-old seedlings grown under short days. Error bars represent the standard error of the mean from three independent experiments ( $n=30$, Student's $t$-test, ${ }^{\star \star} P<0.01$ ). (f) The cell length in PIN8OX lines is more than two times longer than that in the control (see the quantification data in Supplementary Table S2). The arrows indicate the intercellular space. Scale bar, $10 \mu \mathrm{m}$.

localization was, similar to that of PIN5 (ref. 24), insensitive to the treatment with the vesicle trafficking inhibitor brefeldin A (Supplementary Fig. S4). These results strongly suggest that PIN8, similar to PIN5, localizes to the ER and that these proteins might have related functions.

PIN8 transports auxin and regulates cellular auxin homoeostasis. The ER-localized PIN5 was found, in contrast to PM-localized PIN proteins, not to act in intercellular auxin transport, but to be involved in regulating cellular auxin homoeostasis, presumably by compartmentalizing auxin between the ER and the cytosol $^{24}$. Therefore, we tested the role of PIN8 in regulating auxin homoeostasis. The visualization of auxin responses with the auxinresponsive reporter $D R 5:: G U S^{29}$ revealed no changes in pins mutants (data not shown), consistent with a very low expression of PIN8 in sporophytic tissues. In contrast, PIN8OX lines showed a markedly increased DR5 activity, both in the root and in the aerial parts of the seedling (Fig. 4a) suggesting elevated auxin levels.

To directly test the role of PIN8 in regulating auxin levels, we measured free auxin (indole-3-acetic acid (IAA)) levels in the pin 8 mutant and PIN8OX lines. Unfortunately, free IAA measurements directly in pollen were technically impossible, therefore, we used extraction and gas chromatography/mass spectroscopy detection of IAA in root tips, hypocotyls and rosette leaves. These measurements revealed that loss-of-function pin 8 mutants had similar levels 

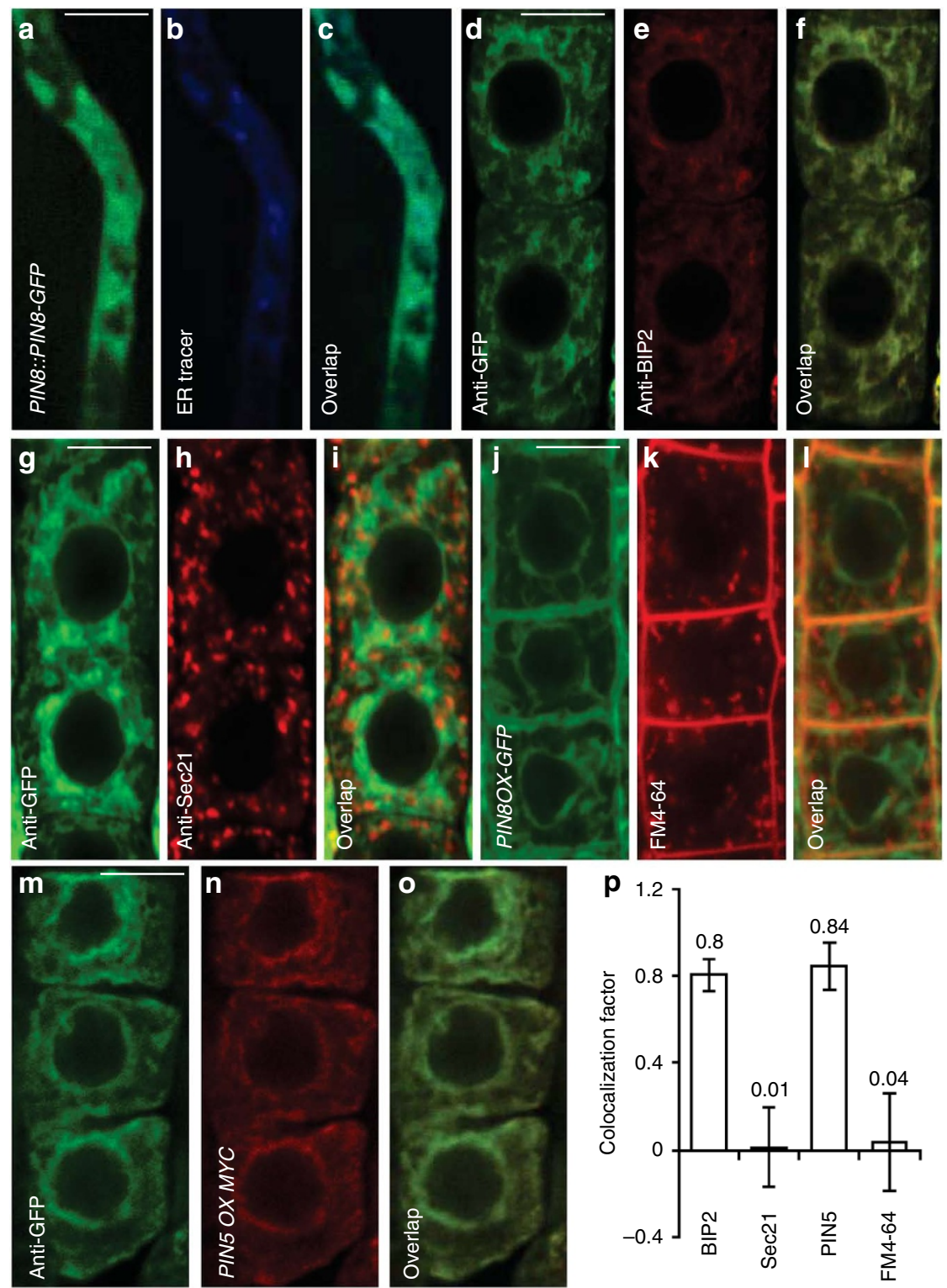

Figure $\mathbf{3}$ | PIN8 localizes to the ER. (a-c) PIN8 localization in the pollen tube of PIN8 ::PIN8-GFP line and co-localization of PIN8-GFP (green) with the ER-tracker dye (blue). (d-f) PIN8 localization in the root of 35S ::PIN8-GFP line and co-localization of PIN8-GFP (green) with ER marker BIP2 staining (red). (g-i) PIN8-GFP (green) does not co-localize with Golgi apparatus (GA) marker Sec21 (red). (j-I) PIN8-GFP (green) does not co-localize with endosomes stained with FM4-64 traced (red). (m-o) PIN8-GFP (green) and PIN5-myc (red) co-localize at the ER. (p) The quantification of PIN8 co-localization with ER (anti-BIP2), GA (anti-Sec21), PIN5 (anti-myc) and endosome (FM4-64 staining) markers confirms localization of PIN8 to the ER but not to the GA, endosomes or plasma membrane. The co-localization factor (the highest is 1.0) was calculated by Zeiss software. For microscope observation, 3-day-old seedlings $(n=6)$ and at least five areas were analysed for each seedling. Scale bar, $5 \mu \mathrm{m}$. Error bars represent the standard error. Live cell imaging (a-c, $\mathbf{j}-\mathbf{I})$ and immunostainings $(\mathbf{d}-\mathbf{f}, \mathbf{g}-\mathbf{i}, \mathbf{m}-\mathbf{0})$.

of free auxin as the control (Fig. 4b), whereas PIN8OX lines showed strongly increased free IAA levels in root tips, hypocotyls and rosette leaves (Fig. 4b). Thus, both DR5-monitored auxin response and direct auxin measurements revealed overall elevated auxin levels in plants overexpressing PIN8.

Next, we quantified the auxin efflux from mesophyll protoplasts isolated from rosette leaves of PIN8 loss-of-function and overexpression lines. Consistent with the changes in auxin levels and PIN8 expression data, protoplasts prepared from pin 8 mutants exhibited a comparable IAA and NAA efflux as wild-type controls, whereas in protoplasts isolated from PIN8OX, the efflux of IAA and NAA was increased (Fig. $4 \mathrm{c}$ and Supplementary Fig. S5), suggesting the auxin transport competence of PIN8. The ER localization of PIN8 in protoplasts (Supplementary Fig. S6) is also consistent with a notion that PIN8 increases efflux of auxin from the ER into the cytoplasm and then presumably out of the protoplast.

To directly assess the ability of PIN8 at the ER membranes, we grew PIN8OX-etiolated plants in liquid culture and ER-enriched membrane fractions were prepared using sucrose gradient centrifugation as described ${ }^{30}$. This fraction was well separated from non-ER fractions including Golgi membranes and other organelles. PIN8GFP was detected in the ER-enriched fraction with an anti-GFP antibody, but no PIN1 was detected with anti-PIN1 (not shown) confirming no or very low PM contamination. Transport assays with 

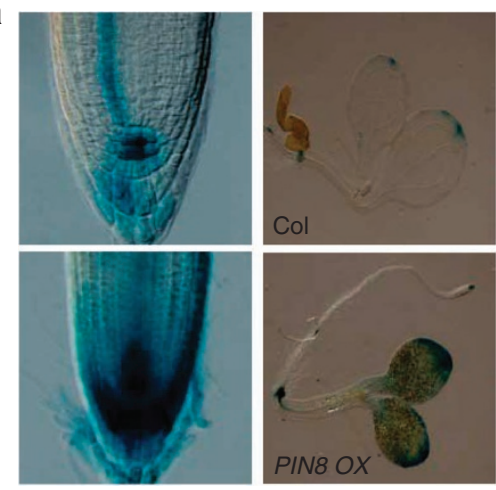

C

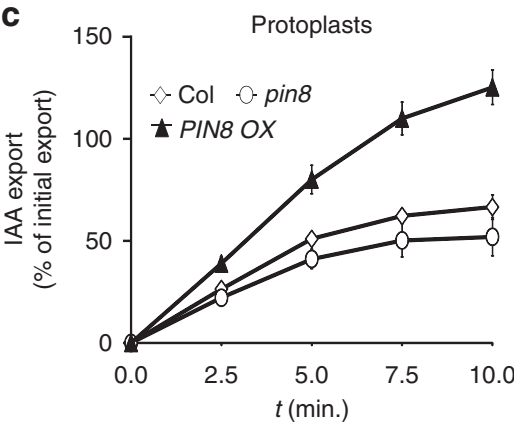

e

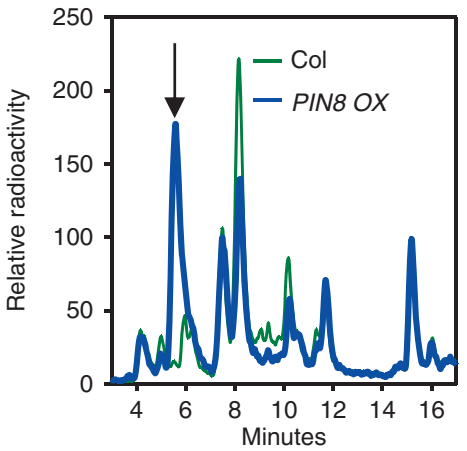

b
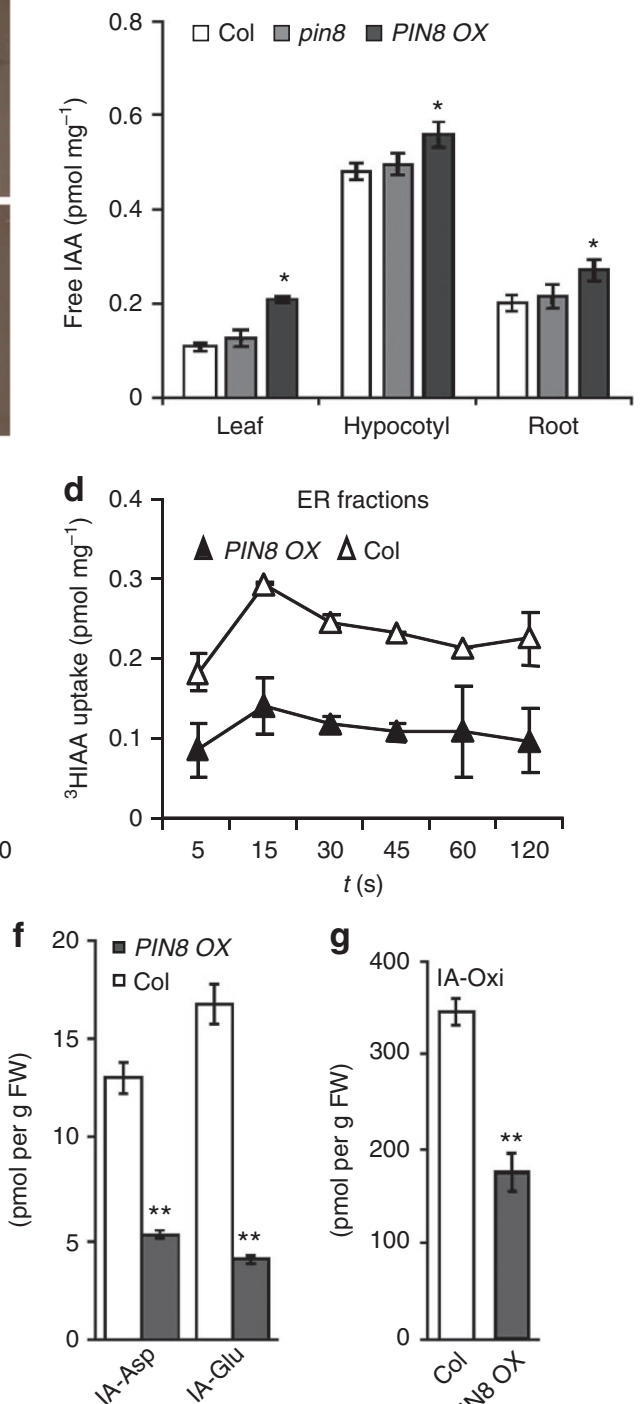

Figure 4 | PIN8 regulates cellular auxin homoeostasis and auxin transport. (a) PIN8OX exhibits enhanced auxin response as shown by the increased DR5::GUS activity in the root and in the aerial parts of the seedling. (b) Changes in free IAA levels in PIN8OX in young leaves/cotyledons, hypocotyls and root tips. Error bars represent the standard error of the mean, $n=5$ (Student's t-test, ${ }^{\star} P<0.05$ ). (c) Protoplasts of $P I N 80 X$ showed a higher rate of IAA export. Error bars represent the standard error of the mean, $n=3$. (d) ${ }^{3} \mathrm{H}$-IAA uptake time course in ER-enriched membrane fractions from Col and PIN8OX plants. Transport assays were performed as described in Methods. ER-enriched membranes were incubated in 50 nM ${ }^{3} \mathrm{H}-\mathrm{IAA}$ fractions. Aliquots were taken at different times, filtered and washed. The radioactivity present in the filters was estimated by liquid scintillation counting and expressed as pmol of ${ }^{3} \mathrm{H}-\mathrm{IAA} / \mathrm{mg}$ of total proteins. The experiment was performed two biological repeats, and the error bars represent the standard error of the mean. (e) The HPLC chromatogram of IAA metabolic profile changes noticeably in the PIN8OX line compared with the wild-type control (2.5-h incubation with ${ }^{3} \mathrm{H}$-IAA). (f,g) Liquid chromatography/mass spectrometry detected strongly decreased levels of IAA conjugates (indole-3-acetyl-aspartate, IA-Asp; indole-3-acetyl-glutamate, IA-Glu) (f), and oxindole-3-acetic acid (OxIAA) (g) in PIN8OX line. White and grey column represent Col and PIN8OX, respectively. Error bars represent the standard error of the mean, $n=3$ (Student's $t$-test, ${ }^{\star \star} P<0.01$ ). FW, fresh weigh.

radiolabelled IAA showed increased auxin accumulation capacity of the ER fractions from the PIN8OX lines as compared with the control (Fig. 4d). In addition, competitive uptake assays with ER-enriched fraction from PIN8OX revealed that synthetic auxins NAA,2,4-D and auxin analogue indole-3-butyric acid can compete with radiolabelled IAA uptake, whereas the biologically inactive analogue benzoic acid ( $1 \mathrm{mM}$ ) did not compete with PIN8-mediated uptake of radiolabelled IAA (Supplementary Fig. S7a). Our auxin transport assays in mesophyll protoplasts from transiently transfected nicotiana benthamiana also indicated that IAA, not benzoic acid, was the preferred substrate of PIN8 action (Supplementary Fig. S7b).
These results show that IAA and other biologically active auxins are preferred substrates of PIN8 action. In an independent set of experiments, lighter ER membranes from PIN8OX seedlings, exhibited acidification after addition of ATP and accumulation of ${ }^{3} \mathrm{H}$-IAA in short-term (60 s) uptake assays, whereas denser ER vesicles, tonoplast vesicles and PM vesicles did not show auxin transport activity (Supplementary Fig. S8). The overall increase in auxin responses and free auxin levels in PIN8OX as well as changed auxin transport capacity of PIN8OX protoplasts and ER-derived membranes altogether suggested that PIN8 is an ER-localized auxin transporter involving in the regulation of auxin homoeostasis. 

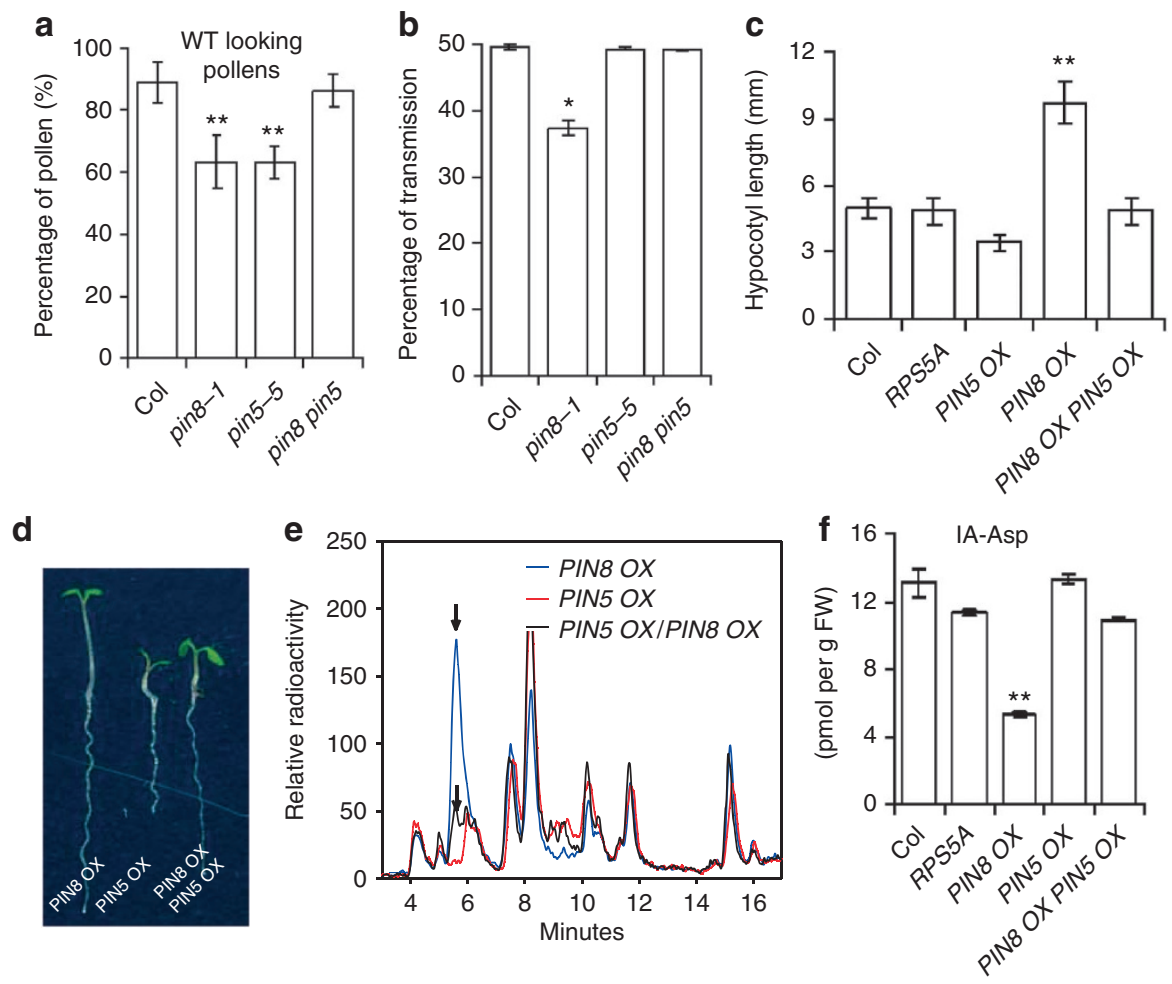

Figure 5 | PIN8 and PIN5 act antagonistically. (a) Morphological defects in pollen of pin5 $(2,400)$ and pin8 $(2,400)$ single mutants were rescued in pin5 pin8 (3,300) double mutants as shown by DAPI staining. A total of 7,100 wild-type (WT) pollens were counted as the control. Error bars represent the standard error of more than 23 independent plants (Student's $t$-test, ${ }^{\star \star} P<0.01$ ). (b) The reduced pollen transmission ability in the pin8 mutant was rescued in the pin 5 pin 8 double mutant. Error bars represent the standard error of more than ten independent crosses (Student's $t$-test, ${ }^{\star} P<0.5$ ). (c,d) The long hypocotyl phenotype of the PIN80X line was rescued in the PIN80X PIN50X line. Error bars represent the standard error of three independent experiments $\left(n=60\right.$, Student's $t$-test, $\left.{ }^{\star \star} P<0.01\right)$. (e) Specific HPLC chromatogram of IAA metabolic profile of PIN8OX was largely rescued in the PIN8OX PIN5OX line (2.5-h incubation with $\left.{ }^{3} \mathrm{H}-I A A\right)$. Arrows indicate the notable changes in the IAA metabolic profile. (f) The decreased IA-Asp of the PIN80X line was rescued in the PIN80X PIN50X line. Error bars represent the standard error of the mean, $n=3$ (Student's $t$-test, ${ }^{\star} P<0.05$ ).

To assess the role of PIN8 in IAA metabolism, we analysed the IAA metabolic profile of PIN8OX transgenic seedlings through high-performance liquid chromatography (HPLC). PIN8 overexpression remodelled the IAA metabolism as demonstrated by the pronounced changes in the HPLC profile (Fig. 4e). Direct analysis by ultra-high-performance liquid chromatography coupled to tandem mass detection of the selected IAA conjugates confirmed the changes in the IAA metabolic profile and showed that the PIN8OX line had a decreased capacity to produce amino-acid conjugates, such as IAAsp (indole-3-acetyl-aspartate), IA-Glu (indole-3-acetyl-glutamate) or OxIAA (oxindole-3-acetic acid; Fig. 4f,g). These results demonstrated that the ER-localized PIN8 auxin transporter, similar to PIN5, is also involved in the regulation of auxin homoeostasis.

PIN8 and PIN5 act antagonistically. To gain additional insights into the roles of PIN5 and PIN8, we systematically compared the loss-of-function and overexpression lines and also generated the pin5 pins double mutants and the PIN8OX PIN5OX double transgenic plants. Similar to pin8, pin5 was also defective in pollen morphology (Figs 1b, 5a), but, in contrast to the single pin5 mutant, the pin 5 pin 8 double mutant largely rescued the pollen morphology defects and the pin 8 defect in transmission through the male gametophyte (Fig. 5a,b). Thus, the pin 5 and pin 8 loss-of-function mutants compensated to a large extent each other in male gametophyte development and function.

Regarding the overexpression lines, PIN5OX seedlings had shorter hypocotyls ${ }^{24}$, whereas PIN8OX seedlings had strikingly longer hypocotyls than the wild-type control (Fig. 5c,d). These overexpression effects were also rescued in the PIN8OX PIN5OX double overexpressing line (Fig. 5c,d). In addition, we also observed that pin 5 significantly enhanced the long hypocotyl phenotype of PIN8OX further supporting the antagonistic roles of PIN5 and PIN8 (Supplementary Fig. S9a). The same could be observed for the flowering time. Under long-day conditions, PIN8OX plants flowered earlier than the control, whereas PIN5OX plants flowered significantly later (Supplementary Fig. S9b,c) and the PIN8OX PIN5OX plants flowered at a time that was comparable to the control plants (Supplementary Fig. S9b,c). These different observations are also consistent with previous observations on the opposite action of PIN5 and PIN8 in root hair development ${ }^{31}$. Thus, both the lossof-function mutants and overexpression lines of PIN5 and PIN8 can largely compensate each other in regulating different developmental processes, suggesting antagonistic roles of these two ER-localized PIN proteins.

Next, we tested genetic interaction between PIN5 and PIN8 in regulating auxin homoeostasis. In the PIN8OX line, both free IAA measurements and enhanced DR5::GUS activity consistently suggested increased free IAA levels (Fig. 4), whereas PIN5OX had been shown to have decreased auxin levels ${ }^{24}$. To test the possible antagonistic roles of PIN5 and PIN8 in the regulation of auxin metabolism, we measured free IAA levels in PIN8OX PIN5OX double transformants. This analysis revealed that, similar to morphological phenotypes, the increased free IAA levels in PIN8OX were largely rescued in the PIN8OX PIN5OX line that exhibited free IAA levels 
comparable to those of the control lines (Supplementary Fig. S10a). The increased IAA export in protoplasts prepared from PIN8 OX also phenocopies pin 5 mutant (Supplementary Fig. S10b). Moreover, notable changes in the IAA metabolic profile of PIN8OX seedlings as demonstrated by the HPLC spectrum were also largely rescued in PIN8OX PIN5OX lines (Fig. 5e). Accordingly, liquid chromatography-tandem mass spectroscopeanalysis confirmed that the decreased capacity of PIN8OX seedlings to conjugate IAA to amino acids was rescued in the PIN8OX PIN5OX lines (Fig. 5f).

Overall, the opposite and mutual compensatory effects of pin 5 and pin 8 loss-of-function and overexpression alleles on male gametophyte and sporophyte phenotypes as well as on auxin homoeostasis and metabolism revealed that PIN5 and PIN8 act antagonistically. This strongly suggests that PIN5 and PIN8 localized both at the same subcellular compartment (ER) can have distinct roles.

\section{Discussion}

In conclusion, this study identified auxin transporter PIN8 with a strong expression in a male gametophyte and revealed a role for auxin transport in regulating pollen development and function. Our results, including localization, auxin transport as well as genetic and physiological analyses, showed that, although both PIN8 and PIN5 auxin transporters are localized at the ER, they can have antagonistic roles in regulating gametophyte and sporophyte development, cellular auxin homoeostasis and metabolism. PIN5 has been proposed to transport auxin intracellularly from the cytoplasm into the ER, where enzymes involved in IAA metabolism are compartmentalized $^{6}$, thus reducing the auxin availability for the PM-based auxin efflux ${ }^{24}$. It is unclear how PIN8 localized to the same intracellular compartment, namely ER, can act antagonistically but it suggests more complex functional interaction between PIN proteins than anticipated so far. A possible scenario would envision transient or more stable interaction of PIN5 and PIN8 negatively regulating each other transport capabilities. Notably, PIN8 activity in Arabidopsis is required only in male gametophyte. It is known that developing and germinating pollen has high levels of auxin ${ }^{32}$, but the auxin role there is unclear. Thus, it is possible that specifically during pollen development, the ER-localized PIN transporters regulate the release of auxin from the internal stores in the ER to drive auxin-mediated pollen tube elongation.

The finding that some PIN proteins localize to the ER and other to the PM brings about an interesting question, namely, which of these functions and cellular localizations is ancestral and which physiological and developmental role did this ancestral PIN protein play. In the moss Physcomitrella, the most typical member of PIN clade localizes to ER, when expressed in BY-2 tobacco cells ${ }^{24}$. As in most ancestral land plants, the gametophyte generation is predominant, the male gametophytic PIN8 might represent a more ancestral form of auxin transporters that were at the ER involved in regulating subcellular auxin homoeostasis, before they acquired PM localization and new function in mediating auxin transport between cells for mediating development of higher plants. Nonetheless, also in higher plants, different ER-localized PIN proteins, including Arabidopsis PIN5, PIN8 and possibly also PIN6, have spatially and/or temporally distinct expression pattern ${ }^{24}$ that can fine-tune the cellular free IAA levels optimal for plant growth and reproduction.

\section{Methods}

Plant material and DNA constructs. For all experiments, we used Arabidopsis thaliana ecotype Columbia (Col). Insertion mutant lines were pin5-5 (ref. 24), pin8-1 (Salk_107965) and pin8-2 (Salk_044651). Transgenic lines were DR5 ::GUS ${ }^{29}$, DR5rev $:$ GFP $P^{33}$, RPS5A $:: G A L 4$ (ref. 34) and RPS5A > > PIN5-myc $\left(\right.$ PIN5 OX) ${ }^{24}$. pin 5 pin 8 double mutants and the PIN8OX PIN5OX double transgenic plants were generated through crossing pin 5-5 with pin8-1 or crossing PIN8OX with PIN5OX. All SALK lines were obtained from the Nottingham Arabidopsis Stock Center. The 35S ::PIN8-GFP (PIN8 OX) line was generated by transformation of the ecotype Columbia (Col) with the 35S ::PIN8-GFP (PIN8OX) construct ${ }^{24}$. The LAT52APIN8 line was generated via replacing the $35 \mathrm{~S}$ promoter with the LAT52 promoter and transformed to the Colecotype. The primers used for cloning the LAT52 promoter are described in Supplementary Table S3.

Growth conditions. Seeds were sterilized with chlorine gas and stratified at $4{ }^{\circ} \mathrm{C}$ for 3 days in the dark. Seedlings were grown vertically on half Murashige and Skoog (MS) medium supplemented with $1 \%$ sucrose and respective drugs. Drugs were purchased from Sigma-Aldrich. Plants were grown under the stable long-day $\left(16 \mathrm{~h}\right.$ light $/ 8 \mathrm{~h}$ dark) or short-day $\left(8 \mathrm{~h} \mathrm{light} / 16 \mathrm{~h}\right.$ dark) conditions at $19^{\circ} \mathrm{C}$ in growth chambers.

Phenotype analyses and GUS ( $\beta$-glucuronidase) staining. Plates were scanned on a flat-bed scanner and hypocotyl lengths were measured with the ImageJ (http://rsb.info.nih.gov/ij/) software. GUS staining was done as described ${ }^{35}$

Microscopy analysis. The immunological analyses were done as previously described $^{36}$. Details on antibodies and dilutions can be found in the later section. GFP samples were scanned without fixation. For confocal microscopy images, Zeiss LSM 510 or Olympus FV10 ASW confocal scanning microscopes were used.

Pollen transmission assays. We used the PIN3 ::PIN3-GFP line for the transmission wild-type controls. Pollen from the hetero PIN3 ::PIN3-GFP, hetero pin8-1 or hetero LAT52::PIN8 was used as a pollen donor, and crossed with Col female. Wild-type control-here is the hetero PIN3 ::PIN3-GFP line-showing around $50 \%$ transmission that was confirmed via antibiotic selection of the resulted seedlings. pin8-1and LAT52::PIN8 transmission was assessed by PCR analysis or antibiotic selection of the resulted seedlings.

Auxin measurements and transport assays. Free IAA measurements and IAA metabolic profiling were done as described ${ }^{24}$. For protoplast transport assays, protoplasts prepared from loss-of-function mutant and overexpression lines were loaded under controlled conditions (loading is performed on ice in order to minimize transport processes with identical amounts of cells and radioactivity) leading to highly comparable loading of cells ${ }^{22}$. Loaded cells were then temperature shifted to $25^{\circ} \mathrm{C}$ enabling catalysed auxin transport (of course both over the ER and PM membrane), and after defined time-points supernatants (containing effluxed radioactivity) were separated from cells by silicon oil centrifugation and quantified. For IAA conjugate quantification, $\sim 10 \mathrm{mg}$ of plant material was taken for analysis. The samples were processed as described ${ }^{37}$ and quantified by ultra-highperformance liquid chromatography coupled to tandem mass detection.

Auxin transport assays in ER-enriched microsomal fractions. Arabidopsis plants grown in liquid culture were homogenized using razor blade in $5 \mathrm{ml}$ of $0.5 \mathrm{M}$ sucrose, $0.1 \mathrm{M} \mathrm{KH}_{2} \mathrm{PO}_{4}$ (pH 6.65), $5 \mathrm{mM} \mathrm{MgCl}_{2}$ and $1 \mathrm{mM}$ dithiothreitol (freshly added). Membranes were separated following the procedure described by Muñoz et al. ${ }^{38,39}$. Briefly, the homogenate was filtered through miracloth (Calbiochem) and centrifuged at $3,000 \mathrm{~g}$ for $3 \mathrm{~min}$. The supernatant was then layered on $5 \mathrm{ml}$ of a $1.3 \mathrm{M}$ sucrose cushion and centrifuged at $108,000 \mathrm{~g}$ for $90 \mathrm{~min}$. The upper phase was removed without disturbing the interface fraction and sucrose layers of 1.1, 0.7 and $0.25 \mathrm{M}$ were overlaid on the membrane pad. The discontinuous sucrose gradient was then centrifuged at $108,000 \mathrm{~g}$ for $90 \mathrm{~min}$. The $1.1 / 1.3 \mathrm{M}$ interface enriched in ER membranes was collected, diluted and centrifuged separately at $108,000 \mathrm{~g}$ for $50 \mathrm{~min}$, the pellet was resuspended in $200 \mu \mathrm{l}$ of $0.5 \mathrm{M}$ sucrose, $0.1 \mathrm{M}$ $\mathrm{KH}_{2} \mathrm{PO}_{4}(\mathrm{pH} 6.65)$ and $5 \mathrm{mM} \mathrm{MgCl}_{2}$ and stored at $-80^{\circ} \mathrm{C}$ until use.

ER-enriched membranes, obtained as described above, were used to perform $\left[{ }^{3} \mathrm{H}\right]-$-IAA uptake assays, based in a filtration method previously described ${ }^{39}$. A total of $50 \mu \mathrm{g}$ of protein from the ER-enriched membrane fraction were resuspended in a buffer containing $250 \mathrm{mM}$ sucrose, $20 \mathrm{mM} \mathrm{KCl}, 25 \mathrm{mM}$ Tris- $\mathrm{HCl}$ $(\mathrm{pH} 7)$. The reaction was initiated by adding $100 \mu \mathrm{l}$ of $50 \mathrm{nM}\left[{ }^{3} \mathrm{H}\right]-\mathrm{IAA}(20 \mathrm{nCi})$, to reach $1 \mathrm{ml}$, final volume. Aliquots were taken at different times and filtered through $0.45-\mu \mathrm{m}$ cellulose-ester filters (Millipore), previously treated with $250 \mathrm{mM}$ sucrose, $20 \mathrm{mM} \mathrm{KCl}, 25 \mathrm{mM}$ Tris- $\mathrm{HCl}(\mathrm{pH} 7)$ and $1 \mathrm{mM}$ IAA. The reaction was stopped by filtering and immediate wash using $5 \mathrm{ml}$ of ice-cold $250 \mathrm{mM}$ sucrose, $20 \mathrm{mM} \mathrm{KCl}, 25 \mathrm{mM}$ Tris- $\mathrm{HCl}$ (pH 7) and $1 \mathrm{mM}$ IAA. The filters were air-dried and the remaining radioactivity was measured in a liquid scintillation counter. The uptake of ${ }^{3} \mathrm{H}$-IAA is reported in ' $\mathrm{nmol}$ of $\left[{ }^{3} \mathrm{H}\right]-\mathrm{IAA} / \mathrm{mg}$ of protein'.

Whole-mount immunolocalization and lifetime confocal microscopy. Wholemount immunological staining on 4-day-old seedlings was done in an Intavis robot. Antibodies were used at the following dilutions: rabbit anti-BIP2 (Hsc70), 1:200 (Stressgen Bioreagents); mouse anti-GFP, 1:600 (Roche); rabbit anti-myc, 1:600 (Sigma-Aldrich). Anti-rabbit and anti-mouse antibodies conjugated with Cy3 or fluorescein isothiocyanate (Dianova, Germany) were used at 1:600 dilutions. For ER-tracker dye labelling, PIN8 ::PIN8-GFP seedlings were mounted in water with a 1:1,000 dilution of ER-tracker dye (Invitrogen). Brefeldin A treatment for $2 \mathrm{~h}$ was performed by incubation of 4-day-old etiolated seedlings on solid MS medium supplemented with brefeldin $\mathrm{A}(50 \mu \mathrm{M})$, counterstaining of cell walls was achieved by mounting seedling roots in $10 \mu \mathrm{M}$ propidium iodide. 
Quantitative PCR analysis. RNA was extracted with the Plant RNeasy kit (Qiagen). Poly(dT) complementary DNA was prepared from flower total RNA. Superscript III reverse transcription (Invitrogen) and quantification were done on a LightCycler 480 apparatus (Roche Diagnostics) with the SYBR Green I Master kit (Roche Diagnostics), according to the manufacturer's instructions. All individual reactions were done in triplicate. Data were analysed as described before ${ }^{40}$. The primers used to quantify gene expression levels are provided in Supplementary Table S3.

Light and fluorescent microscopy. For pollen phenotype observations, flowers from pin or Col- 0 plants were collected to GUS buffer $(0.1 \mathrm{M}$ phosphate buffer, pH 7.0; 10 mM EDTA, pH 8.0; 0.1\% triton X-100) supplemented with $100 \mathrm{ng} \mathrm{ml}^{-1}$ DAPI as described ${ }^{41}$. After 30-min incubation at room temperature in the dark, samples were analysed by bright-field and fluorescence microscopy with Nikon TE2000-E microscope (objective Nikon CFI Plan Fluor ELWD 10×/0.60, eyepiece Nikon CFI $10 \times / 22$, intermediate magnification $\times 1-\times 1.5$; Nikon).

Pollen germination in vitro. Pollens were collected from flowers opened day (day 0 ) of Col and pin8-1 plants. Subsequently they were germinated on a germination medium on a microscope slide according to Boavida and McCormick ${ }^{42}$. Pollen germination medium was always prepared fresh from $0.5 \mathrm{M}$ stock solutions of the main components $\left(5 \mathrm{mM} \mathrm{KCl}, 0.01 \% \mathrm{H}_{3} \mathrm{BO}_{3}, 5 \mathrm{mM} \mathrm{CaCl}_{2}, 1 \mathrm{mM} \mathrm{MgSO}_{4}\right.$ ) using autoclaved water. Sucrose $(10 \%)$ was added and $\mathrm{pH}$ was adjusted to 7.5 with $\mathrm{NaOH}$. Low-melting agarose (Amresco, Solon, $\mathrm{OH}$ ) was added to final $1.5 \%$ concentration and melted in a microwave oven. Pollen grains from three flowers were spread on the surface of $250 \mu \mathrm{l}$ agarose germination pads on microscope slides covered by polypropylene foil by inverting the flower with the help of tweezers and gently bringing it onto agarose surface. The whole flower was used as a 'brush' to spread pollen uniformly. The slides were incubated upside down in a moisture incubation chamber for $16 \mathrm{~h}$ in the dark at $22^{\circ} \mathrm{C}$ and $100 \%$ humidity. The samples were examined by bright-field microscopy using Nikon TE2000-E microscope (objective Nikon CFI Plan UW $2 \times / 0.06$, eyepiece Nikon CFI $10 \times / 22$, intermediate magnification $\times 1-\times 1.5$ ). The germinating pollen was defined as a pollen with a clearly visible pollen tube with length at least 1 diameter of pollen grain. The germination was carried out overnight $(16 \mathrm{~h})$. The percentage of germinated pollen was scored manually from captured image. From each microscope slide, five to six individual areas were taken. For NAA pollen germination test, $1 \mu \mathrm{M}$ NAA was dissolved in the germination medium to $100 \mathrm{nM}$ final concentration.

\section{References}

1. Leyser, O. The power of auxin in plants. Plant Physiol. 154, 501-505 (2010).

2. Leyser, O. Auxin distribution and plant pattern formation: how many angels can dance on the point of PIN? Cell 121, 819-822 (2005).

3. Santner, A. \& Estelle, M. Recent advances and emerging trends in plant hormone signalling. Nature 459, 1071-1078 (2009).

4. Kepinski, S. \& Leyser, O. Plant development: auxin in loops. Curr. Biol. 15, R208-210 (2005).

5. Chapman, E. J. \& Estelle, M. Mechanism of auxin-regulated gene expression in plants. Annu. Rev. Genet. 43, 265-285 (2009).

6. Woodward, A. W. \& Bartel, B. Auxin: regulation, action, and interaction. Ann. Bot. 95, 707-735 (2005).

7. Grunewald, W. \& Friml, J. The march of the PINs: developmental plasticity by dynamic polar targeting in plant cells. EMBO J. 29, 2700-2714 (2010).

8. Vanneste, S. \& Friml, J. Auxin: a trigger for change in plant development. Cell 136, 1005-1016 (2009).

9. Pagnussat, G. C., Alandete-Saez, M., Bowman, J. L. \& Sundaresan, V. Auxindependent patterning and gamete specification in the Arabidopsis female gametophyte. Science 324, 1684-1689 (2009)

10. Wu, M.- F., Tian, Q. \& Reed, J. W. Arabidopsis microRNA167 controls patterns of ARF6 and ARF8 expression, and regulates both female and male reproduction. Development 133, 4211-4218 (2006).

11. Feng, X.- L. et al. Auxin flow in anther filaments is critical for pollen grain development through regulating pollen mitosis. Plant Mol. Biol. 61, 215-226 (2006).

12. Cecchetti, V., Altamura, M. M., Falasca, G., Costantino, P. \& Cardarelli, M. Auxin regulates Arabidopsis anther dehiscence, pollen maturation, and filament elongation. Plant Cell 20, 1760-1774 (2008).

13. Chen, D. \& Zhao, J. Free IAA in stigmas and styles during pollen germination and pollen tube growth of Nicotiana tabacum. Physiol. Plant 134, 202-215 (2008).

14. Zhao, Y. Auxin biosynthesis and its role in plant development. Annu. Rev. Plant Biol. 61, 49-64 (2010)

15. Stepanova, A. N. et al. TAA1-mediated auxin biosynthesis is essential for hormone crosstalk and plant development. Cell 133, 177-191 (2008).

16. Ikeda, Y. et al. Local auxin biosynthesis modulates gradient-directed planar polarity in Arabidopsis. Nat. Cell Biol. 11, 731-8 (2008).

17. Petrášek, J. \& Friml, J. Auxin transport routes in plant development. Development 136, 2675-2688 (2009).
18. Swarup, R. \& Bennett, M. Auxin transport: the fountain of life in plants? Dev. Cell 5, 824-826 (2003).

19. Swarup, K. et al. The auxin influx carrier LAX3 promotes lateral root emergence. Nat. Cell Biol. 10, 946-954 (2008).

20. Petrášek, J. et al. PIN proteins perform a rate-limiting function in cellular auxin efflux. Science 312, 914-918 (2006).

21. Noh, B., Murphy, A. S. \& Spalding, E. P. Multidrug resistance-like genes of Arabidopsis required for auxin transport and auxin-mediated development. Plant Cell 13, 2441-2454 (2001).

22. Geisler, M. et al. Cellular efflux of auxin catalyzed by the Arabidopsis MDR/ PGP transporter AtPGP1. Plant J. 44, 179-94 (2005).

23. Křeček, P. et al. The PIN-FORMED (PIN) protein family of auxin transporters. Genome Biol. 10, 249-256 (2009).

24. Mravec, J. et al. Subcellular homeostasis of phytohormone auxin is mediated by the ER-localized PIN5 transporter. Nature 459, 1136-1140 (2009).

25. Dupáková, N. et al. Arabidopsis Gene Family Profiler (aGFP)-user-oriented transcriptomic database with easy-to-use graphic interface. BMC Plant Biol. 7, 39: 10.1186/1471-2229-7-39 (2007).

26. Peer, W. A. et al. Mutation of the membrane-associated M1 proteaseAPM1 results in distinct embryonic and seedling developmental defects in Arabidopsis. Plant Cell 21, 1693-1721 (2009).

27. Sánchez-Morán, E. et al. A puromycin-sensitive aminopeptidase is essential for meiosis in Arabidopsis thaliana. Plant Cell 16, 2895-909 (2004).

28. Ishikawa, F., Suga, S., Uemura, T., Sato, M. H. \& Maeshima, M. Novel type aquaporin SIPs are mainly localized to the ER membrane and show cell-specific expression in Arabidopsis thaliana. FEBS Lett. 579, 5814-5820 (2005).

29. Ulmasov, T., Liu, Z. B., Hagen, G. \& Guilfoyle, T. J. Composite structure of auxin response elements. Plant Cell 7, 1611-1623 (1995).

30. Reyes, F., León, G., Donoso, M., Brandizzí, F., Weber, A. P. \& Orellana, A. The nucleotide sugar transporters AtUTrl and AtUTr3 are required for the incorporation of UDP-glucose into the endoplasmic reticulum, are essential for pollen development and are needed for embryo sac progress in Arabidopsis thaliana. Plant J. 61, 423-35 (2010).

31. Ganguly, A. et al. Differential auxin-transporting activities of PIN-FORMED proteins in Arabidopsis root hair cells. Plant Physiol. 153, 1046-1061 (2010).

32. Aloni, R., Aloni, E., Langhans, M. \& Ullrich, C. I. Role of auxin in regulating Arabidopsis flower development. Planta 223, 315-328 (2006).

33. Friml, J. et al. Efflux-dependent auxin gradients establish the apical-basal axis of Arabidopsis. Nature 426, 147-153 (2003)

34. Weijers, D. et al. Maintenance of embryonic auxin distribution for apical-basal patterning by PIN-FORMED-dependent auxin transport in Arabidopsis. Plant Cell 17, 2517-2526 (2005).

35. Benková, E. et al. Local, efflux-dependent auxin gradients as a common module for plant organ formation. Cell 115, 591-602 (2003).

36. Sauer, M., Paciorek, T., Benková, E. \& Friml, J. Immunocytochemical techniques for whole mount in situ protein localization in plants. Nat. Protoc. 1, 98-103 (2006).

37. Pencik, A. et al. Isolation of novel indole-3-acetic acid conjugates by immunoaffinity extraction. Talanta 80, 651-655 (2009).

38. Munoz, P., Norambuena, L. \& Orellana, A. Evidence for a UDP-glucose transporter in Golgi apparatus-derived vesicles from pea and its possible role in polysaccharide biosynthesis. Plant Physiol. 112, 1585-1594 (1996).

39. Liang, F. \& Sze, H. A high-affinity Ca2+ pump, ECA1, from the endoplasmic reticulum is inhibited by cyclopiazonic acid but not by thapsigargin. Plant Physiol. 118, 817-825 (1998).

40. Ding, Z. J. et al. Auxin regulates distal stem cell differentiation in Arabidopsis roots. Proc. Natl Acad. Sci. USA 107, 12046-120 (2010).

41. Park, S. K., Howden, R. \& Twell, D. The Arabidopsis thaliana gametophytic mutation gemini pollen disrupts microspore polarity, division asymmetry and pollen cell fate. Development 125, 3789-3799 (1998).

42. Boavida, L. C. \& McCormick, S. Temperature as a determinant factor for increased and reproducible in vitro pollen germination in Arabidopsis thaliana. Plant J. 52, 570-582 (2007).

\section{Acknowledgements}

We thank Vincent Vincenzetti, Laurence Charrier and Jinsheng Zhu for help with yeas and tobacco transport assays; Markéta Pařezová for BY-2 cell line transformations and maintenance; Klára Hoyerová and Petre I. Dobrev for help with metabolic profiling. This work was supported by the funding from the projects CZ.1.07/2.3.00/20.0043 and CZ.1.05/1.1.00/02.0068 (to CEITEC, Central European Institute of Technology) and the Odysseus program of the Research Foundation-Flanders to J.F., from 'Qilu Scholarship' of Shandong University (11200081963024) to Z.D., from German Science Foundation (DFG-SFB480/A-10) to S.P., from Swiss National Science Foundation (Grant No. 31003A-125001) and Forschungspool of the University of Fribourg to M.G., from the Ministry of Education, Youth and Sports of the Czech Republic, projects LC06034 to P.S., J.P. and E.Z., KAN200380801 to J.R. (Jakub Rolčík), and OC10054 to N.D., from the Czech Science Foundation (522/09/0858) to D.H, ICM-P10-062-F, Basal Program PFB-16, FONDAP-CRG to A.O. and NSF-IOS (0820648) to A.S.M. 


\section{Author contributions}

J.F., Z.D., A.M. and M.G. planned experiments. B.W., N.D., S.S., P.S., A.P., S.P., J.M., X.C., I.M., N.C., J.R. (Jesica Reemmer) and Z.D. performed experiments. J.F., Z.D., M.G., D.H., J.P., J.R. (Jakub Rolčík), X.C., I.M., A.O., R.T. and E.Z. analysed the data. J.F. and Z.D. wrote the paper. All authors discussed the results and commented on the manuscript.

\section{Additional information}

Supplementary Information accompanies this paper at http://www.nature.com/ naturecommunications
Competing financial interests: The authors declare no competing financial interests.

Reprints and permission information is available online at http://npg.nature.com/ reprintsandpermissions/

How to cite this article: Ding, Z. et al. ER-localized auxin transporter PIN8 regulates auxin homoeostasis and male gametophyte development in Arabidopsis. Nat. Commun. 3:941 doi: 10.1038/ncomms1941 (2012).

License: This work is licensed under a Creative Commons Attribution-NonCommercialNoDerivative Works 3.0 Unported License. To view a copy of this license, visit http:// creativecommons.org/licenses/by-nc-nd/3.0/ 


\title{
Corrigendum: ER-localized auxin transporter PIN8 regulates auxin homoeostasis and male gametophyte development in Arabidopsis
}

\author{
Zhaojun Ding, Bangjun Wang, Ignacio Moreno, Nikoleta Dupláková, Sibu Simon, Nicola Carraro, Jesica Reemmer, \\ Aleš Pěnčík, Xu Chen, Ricardo Tejos, Petr Skůpa, Stephan Pollmann, Jozef Mravec, Jan Petrášek, Eva Zažímalová, \\ David Honys, Jakub Rolčík, Angus Murphy, Ariel Orellana, Markus Geisler \& Jiři Friml
}

Nature Communications 3:941 doi: 10.1038/ncomms1941 (2012); Published 3 Jul 2012; Updated 5 Feb 2013

This Article contains errors in Figs 1, 4, and Supplementary Fig. S6, for which we apologize.

In Fig. 1b, the Col picture was inadvertently duplicated from the image below it. In addition, the legend should have defined the scale bar for panel e as $1 \mu \mathrm{m}$. The correct version of Fig. 1 and its legend appears below.
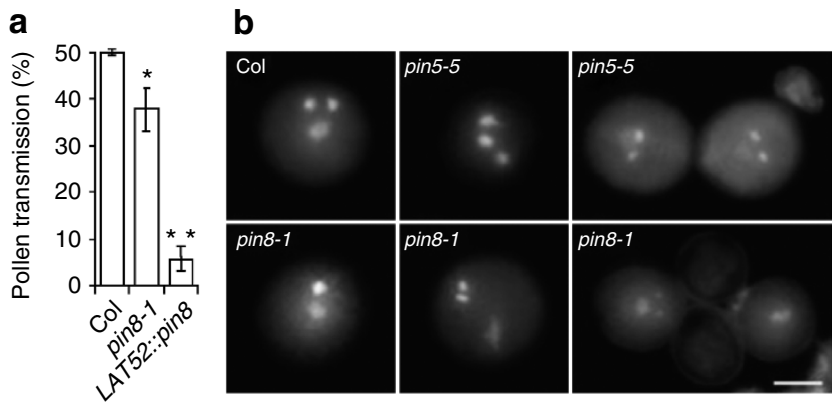
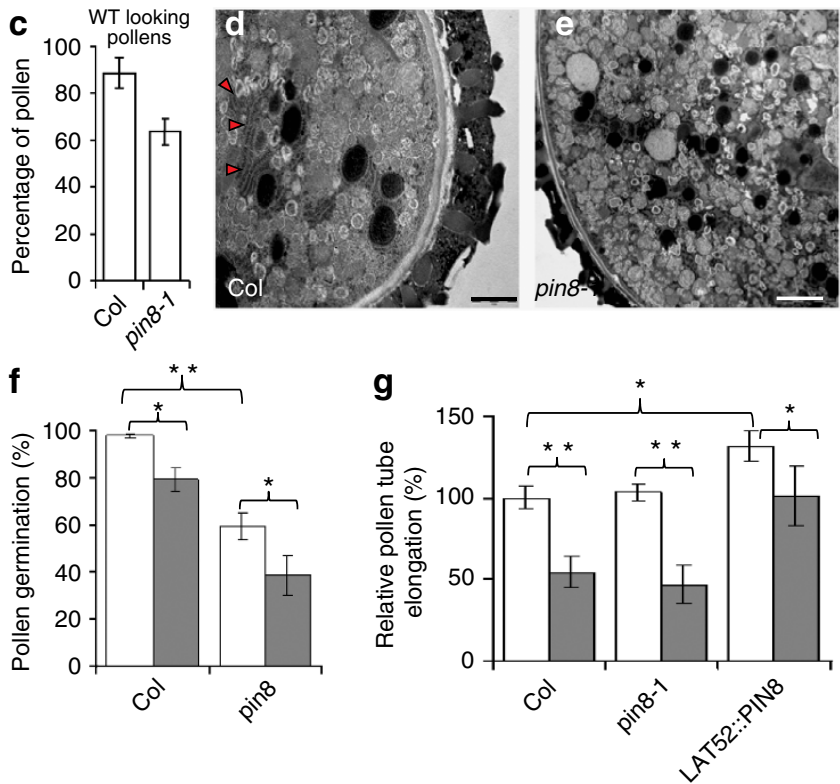

Figure 1 | PIN8 is involved predominantly in the male gametophyte development. (a) The pin8 mutant and the LAT52::PIN8 line showed reduced pollen transmission ability. Error bars represent the standard error of more than ten independent crosses (Student's $t$-test, ${ }^{*} P<0.05$ ). (b,c) DAPI staining analysis showing defects in the morphology of pin8 and pin5 mutant pollen. Both mutants showed distorted and/or misplaced male germ unit and less frequently also exhibit pollen mitosis defects. Sometimes, collapsed pollen grains were observed. Scale bar, $10 \mu \mathrm{m}$. Error bars represent the standard error of more than 23 independent plants (Student's t-test, ${ }^{\star \star} P<0.01$ ). (d,e) Typical ER clusters in wild-type (WT) pollen (d) were not observed in $10-15 \%$ pin 8 pollen (observed 150 pollen grains) (e) by transmission electron microscope analysis. Scale bar, $1 \mu \mathrm{m}$. Red arrows mark ER clusters. (f) pin8 (2256 pollen were analysed) shows reduced in vitro pollen germination abilities (2,814 Col pollens were analysed as the control) and increased sensitivity to auxin treatment ( $100 \mathrm{nM}$ NAA) with a $35 \%$ reduction of pollen germination in pin8 (5,017 pollens were analysed) compared with the $19 \%$ reduction in $\mathrm{Col}(1,131$ pollens were analysed). Error bars represent the standard error of more than ten independent plants (Student's $t$-test, ${ }^{\star} P<0.05$; ${ }^{\star} P P<0.01$ ). White and grey columns represent without and with NAA treatment, respectively. (g) Overexpression of PIN8 in pollen in the LAT52::PIN8 line strongly increased the resistance (with a $24 \%$ inhibition of pollen tube length compared with the $46 \%$ inhibition in $\mathrm{Col}$ ) of in vitro pollen germination to NPA (100 $\mu \mathrm{m}$ NPA) treatment. Error bars represent the standard error of more than six independent plants (Student's $t$-test, $\left.{ }^{\star} P<0.05 ;{ }^{\star} P<0.01\right)$. White and grey column represent without and with NPA treatment, respectively, in Col, pin8 or LAT52::PIN8. 
In Fig. 4d, the filled triangles should have been labelled as $\mathrm{Col}$ and the open triangles as PIN8OX. The correct version of Fig. 4 appears below.

a
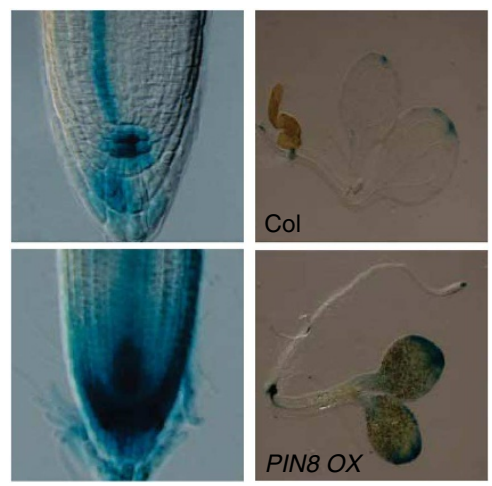

C

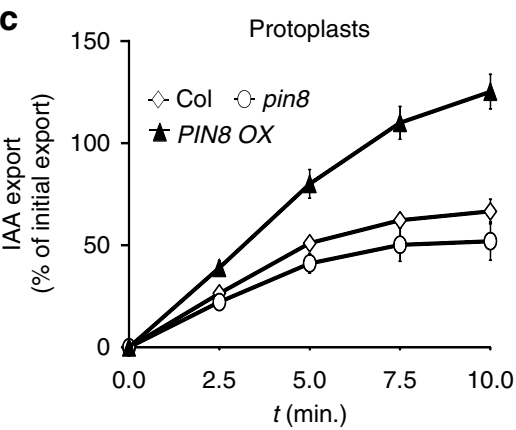

e

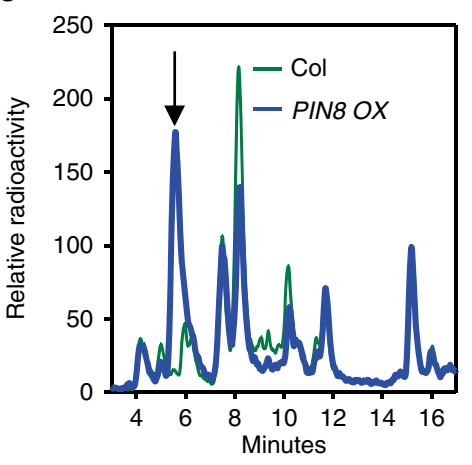

b

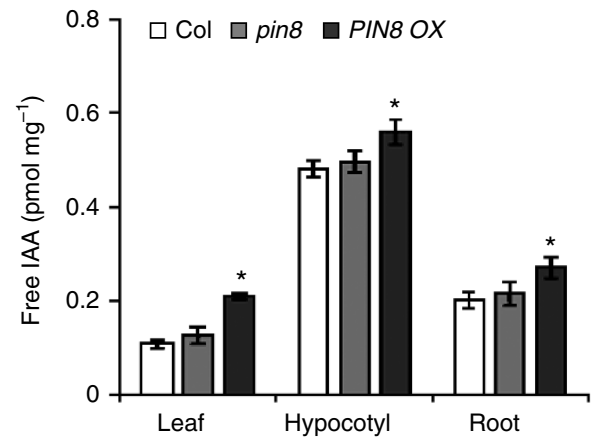

d
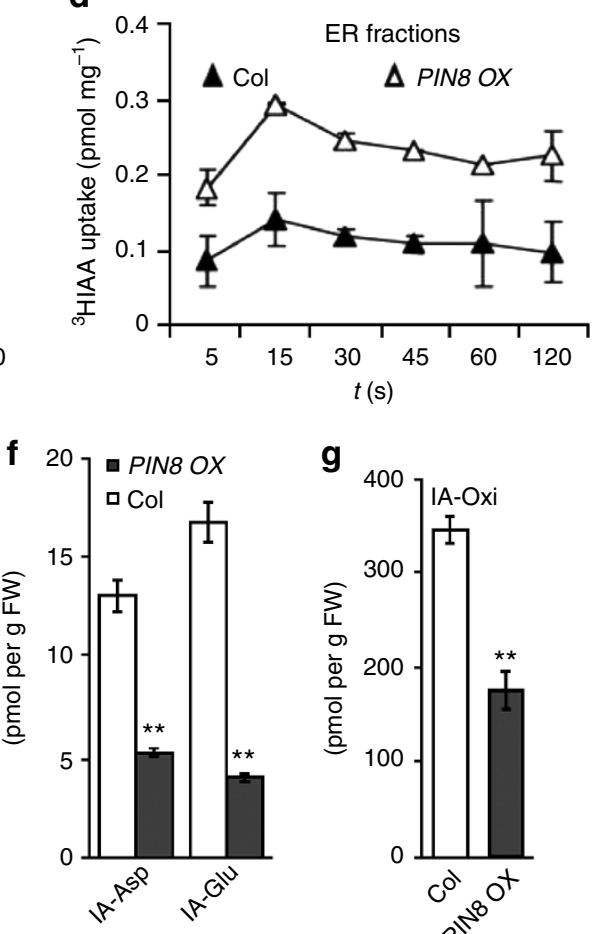

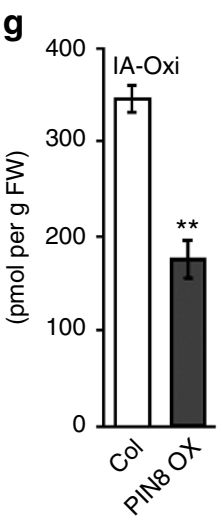

In the legend to Supplementary Fig. S6, the scale bar should be defined as $20 \mu \mathrm{m}$. 